\title{
Fabrication of Large Dual-Polarized Multichroic TES Bolometer Arrays for CMB Measurements with the SPT-3G Camera
}

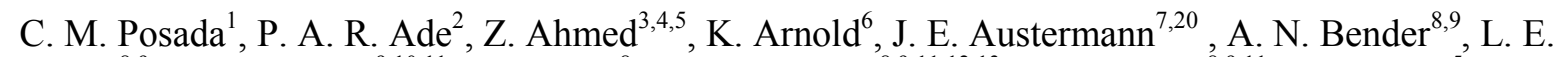 \\ Bleem $^{8,9}$, B. A. Benson ${ }^{9,10,11}$, K. Byrum ${ }^{8}$, J. E. Carlstrom ${ }^{8,9,11,12,13}$, C. L. Chang ${ }^{8,9,11}$, H. M. Cho ${ }^{5}$, S. T. \\ Ciocys $^{8}$, J. F. Cliche ${ }^{14}$, T. M. Crawford ${ }^{9,11}$, A. Cukierman ${ }^{15}$, D. Czaplewski ${ }^{16}$, J. Ding ${ }^{1}$, R. Divan ${ }^{16}$ T. de

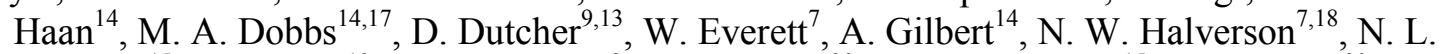 \\ Harrington $^{15}$, K. Hattori ${ }^{19}$, J. W. Henning ${ }^{9}$, G. C. Hilton ${ }^{20}$, W. L. Holzapfel ${ }^{15}$, J. Hubmayr ${ }^{20}$, K. D. \\ Irwin $^{3,4,5}$, O. Jeong ${ }^{15}$, R. Keisler, ${ }^{3,4}$, D. Kubik ${ }^{10}$, C. L. Kuo ${ }^{3,4,5}$, A. T. Lee ${ }^{15,21}$, E. M. Leitch, ${ }^{9,11}$, S. Lendinez ${ }^{1}$, \\ S. S. Meyer ${ }^{911,12,13}$, C. S. Miller ${ }^{16}$, J. Montgomery ${ }^{14}$, M. Myers ${ }^{15}$, A. Nadolski ${ }^{27}$, T. Natoli ${ }^{9,13}$, H.

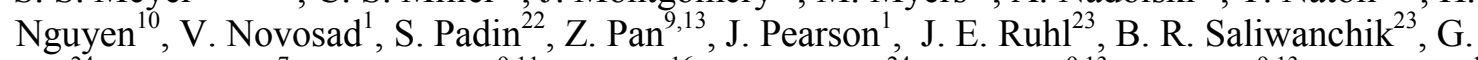 \\ Smecher $^{24}$, J. T. Sayre, E. Shirokoff ${ }^{911}$, L. Stan ${ }^{16}$, A. A. Stark ${ }^{24}$, J. Sobrin ${ }^{9,13}$, K. Story ${ }^{9,13}$, A. Suzuki ${ }^{15}$, K. \\ L. Thompson ${ }^{3,4,5}$, C. Tucker ${ }^{2}$, K. Vanderlinde ${ }^{25,26}$, J. D. Vieira ${ }^{27,28}$, G. Wang ${ }^{8}$, N. Whitehorn ${ }^{15}$, V.

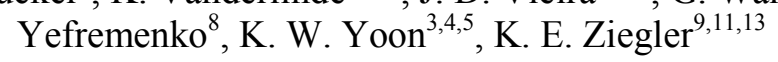
${ }^{1}$ Argonne National Laboratory, Material Science Division, 9700 S. Cass Avenue, Argonne, IL, USA 60439
${ }^{2}$ School of Physics and Astronomy, Cardiff University, Cardiff CF24 3YB, United Kingdom
${ }^{3}$ Kavli Institute for Particle Astrophysics and Cosmology, Stanford University, 452 Lomita Mall, Stanford, CA 94305
${ }^{4}$ Department of Physics, Stanford University, 382 Via Pueblo Mall, Stanford, CA 94305
${ }^{5}$ SLAC National Accelerator Laboratory, 2575 Sand Hill Road, Menlo Park, CA 94025
${ }^{6}$ Department of Physics, University of California, San Diego, CA 92093

${ }^{7}$ CASA, Department of Astrophysical and Planetary Sciences, University of Colorado, Boulder, Colorado 80309

${ }^{8}$ Argonne National Laboratory, High-Energy Physics Division, 9700 S. Cass Avenue, Argonne, IL, USA 60439

${ }^{9}$ Kavli Institute for Cosmological Physics, University of Chicago, 5640 South Ellis Avenue, Chicago, IL 60637 USA

${ }^{10}$ Fermi National Accelerator Laboratory, MS209, P.O. Box 500, Batavia, IL 60510-0500

${ }^{11}$ Department of Astronomy and Astrophysics, University of Chicago, 5640 South Ellis Avenue, Chicago, IL 60637

${ }^{12}$ Enrico Fermi Institute, University of Chicago, 5640 South Ellis Avenue, Chicago, IL 60637

${ }^{13}$ Department of Physics, University of Chicago, 5640 South Ellis Avenue, Chicago, IL 60637

${ }^{14}$ Department of Physics, McGill University, 3600 Rue University, Montreal, Quebec H3A 2T8, Canada

${ }^{15}$ Department of Physics, University of California, Berkeley, CA 94720

${ }^{16}$ Argonne National Laboratory, Center for Nanoscale Materials, 9700 S. Cass Avenue, Argonne, IL, USA 60439

${ }^{17}$ Canadian Institute for Advanced Research, CIFAR Program in Cosmology and Gravity, Toronto, ON, M5G 1Z8, Canada

${ }^{18}$ Department of Physics, University of Colorado, Boulder, CO 80309

${ }^{19}$ High Energy Accelerator Research Organization (KEK), Tsukuba, Ibaraki 305-0801, Japan

${ }^{20}$ NIST Quantum Devices Group, 325 Broadway Mailcode 817.03, Boulder, CO, USA 80305

${ }^{21}$ Physics Division, Lawrence Berkeley National Laboratory, Berkeley, CA 94720

${ }^{22}$ California Institute of Technology, 1200 E. California Blvd., Pasadena, CA 91125

${ }^{23}$ Physics Department, Case Western Reserve University, Cleveland, OH 44106

${ }^{24}$ Harvard-Smithsonian Center for Astrophysics, 60 Garden Street, Cambridge, MA 02138

${ }^{24}$ Three-Speed Logic, Inc., Vancouver, B.C., V6A 2J8, Canada

${ }^{25}$ Dunlap Institute for Astronomy \& Astrophysics, University of Toronto, 50 St George St, Toronto, ON, M5S 3H4, Canada

${ }^{26}$ Department of Astronomy \& Astrophysics, University of Toronto, 50 St George St, Toronto, ON, M5S 3H4, Canada

${ }^{27}$ Astronomy Department, University of Illinois, 1002 W. Green Street, Urbana, IL 61801 USA

${ }^{28}$ Department of Physics, University of Illinois, 1110 W. Green Street, Urbana, IL 61801 USA 


\section{ABSTRACT}

This work presents the procedures used by Argonne National Laboratory to fabricate large arrays of multichroic transition-edge sensor (TES) bolometers for cosmic microwave background (CMB) measurements. These detectors will be assembled into the focal plane for the SPT-3G camera, the third generation CMB camera to be installed in the South Pole Telescope. The complete SPT-3G camera will have approximately 2690 pixels, for a total of 16,140 TES bolometric detectors. Each pixel is comprised of a broad-band sinuous antenna coupled to a $\mathrm{Nb}$ microstrip line. In-line filters are used to define the different band-passes before the millimeterwavelength signal is fed to the respective Ti/Au TES bolometers. There are six TES bolometer detectors per pixel, which allow for measurements of three band-passes $(95 \mathrm{GHz}, 150 \mathrm{GHz}$ and $220 \mathrm{GHz}$ ) and two polarizations. The steps involved in the monolithic fabrication of these detector arrays are presented here in detail. Patterns are defined using a combination of stepper and contact lithography. The misalignment between layers is kept below $200 \mathrm{~nm}$. The overall fabrication involves a total of 16 processes, including reactive and magnetron sputtering, reactive ion etching, inductively coupled plasma etching and chemical etching.

\section{INTRODUCTION}

The cosmic microwave background $(\mathrm{CMB})$ is remnant radiation from the early universe that provides a unique window for exploring fundamental physics. The discovery of the CMB in 1965 [1, 2] validated the Hot Big Bang model of cosmology [3], its homogeneity led to the theory of inflation, and its anisotropy [4] showed the geometry of the universe is flat and gave a complete census of the matter-energy content of the universe [5-8]. Studies of the CMB continue to be a cornerstone of modern cosmology. Current and upcoming CMB experiments are focused on characterizing the extremely faint CMB polarization, particularly the tensor induced " $\mathrm{B}$ mode" component. These measurements have the potential to detect primordial gravitational waves generated during an epoch of inflation. The CMB polarization also contains the imprint of large-scale structure in the universe due to the gravitational lensing of the CMB photons as they travel through space. The growth of large-scale structure is influenced by neutrino mass, therefore $\mathrm{CMB}$ polarization measurements can constrain neutrino physics.

CMB detectors have evolved to the point where their sensitivity is limited by photon noise, which refers to the intrinsic variation of an incident photon flux. Increasing the sensitivity of CMB experiments to a level required for characterizing the CMB B-mode polarization requires fabricating focal planes with orders of magnitude more detectors than current instruments. Multichroic pixels constitute an economic approach to increasing the number of detectors within a given focal plane area. This work presents the fabrication of a large multichroic arrays of superconducting transition-edge sensor (TES) bolometers [9,10,11], which will be deployed in the next generation experiment on the South Pole Telescope, SPT-3G [12].

This paper is structured as follows, we begin with a general description of the SPT-3G focal plane and the multichroic pixel architecture; we then present an overview of the fabrication 
process before providing a detailed description of the fabrication of the individual components of a detector.

\section{SPT-3G FOCAL PLANE AND WAFER ARRAY}

The SPT-3G camera will consists of ten individual hexagonal arrays, which will fill the SPT$3 \mathrm{G}$ focal plane as shown in Figure 1-(a). Figure 1-(b) shows one of the detector array wafers after fabrication is completed. Each hexagonal detector array consists of 271 pixels fabricated from a single $150 \mathrm{~mm}$ silicon wafer. In each wafer, two pixels are used to pattern the alignment marks required during fabrication.

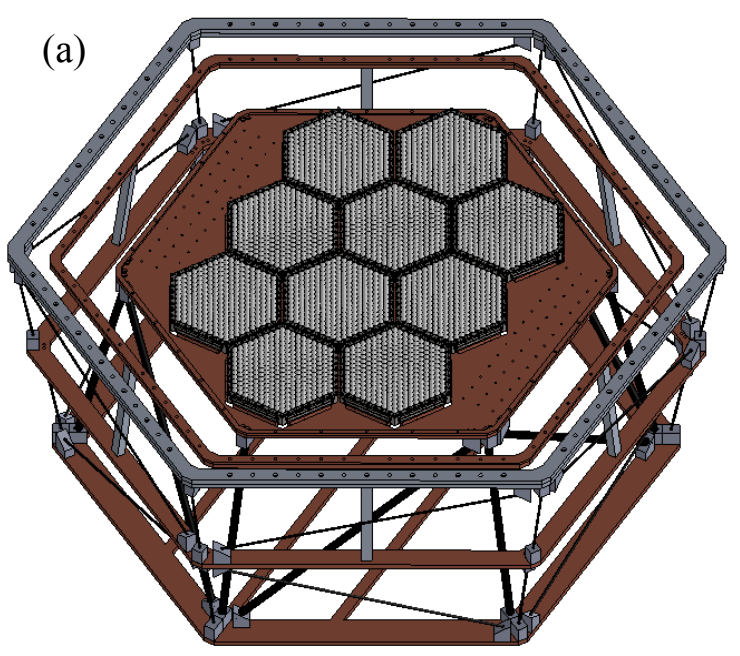

(b)

Figure 1. (a) CAD drawing of the SPT-3G focal plane and support structure. (b) Photograph of a single detector array of 271 pixels after fabrication at Argonne National Laboratory is completed.

Each pixel in the hexagonal array has six detectors, allowing for individual measurement of three spectral bands (centered at $95 \mathrm{GHz}, 150 \mathrm{GHz}$ and $220 \mathrm{GHz}$ ), each in two orthogonal polarizations. In total, the SPT-3G focal plane will be composed of 16140 detectors. $5 \mu$ m wide $\mathrm{Nb}$ traces with a $5 \mu \mathrm{m}$ pitch are routed from each pixel to bond-pads at the edges of the hexagonal array, as shown in Figure 2. Deep access automatic aluminum wire-bonding is used to electrically connect the detectors to the external readout system [13]. The bond-pads are fabricated with a $100 \mu \mathrm{m}$ pitch and are arranged in a staggered T design to allow for manual wire bonding if necessary. A description of the SPT-3G instrument and components, including a description of its optical design, receiver design, cryogenics and readout system is presented elsewhere [12]. 


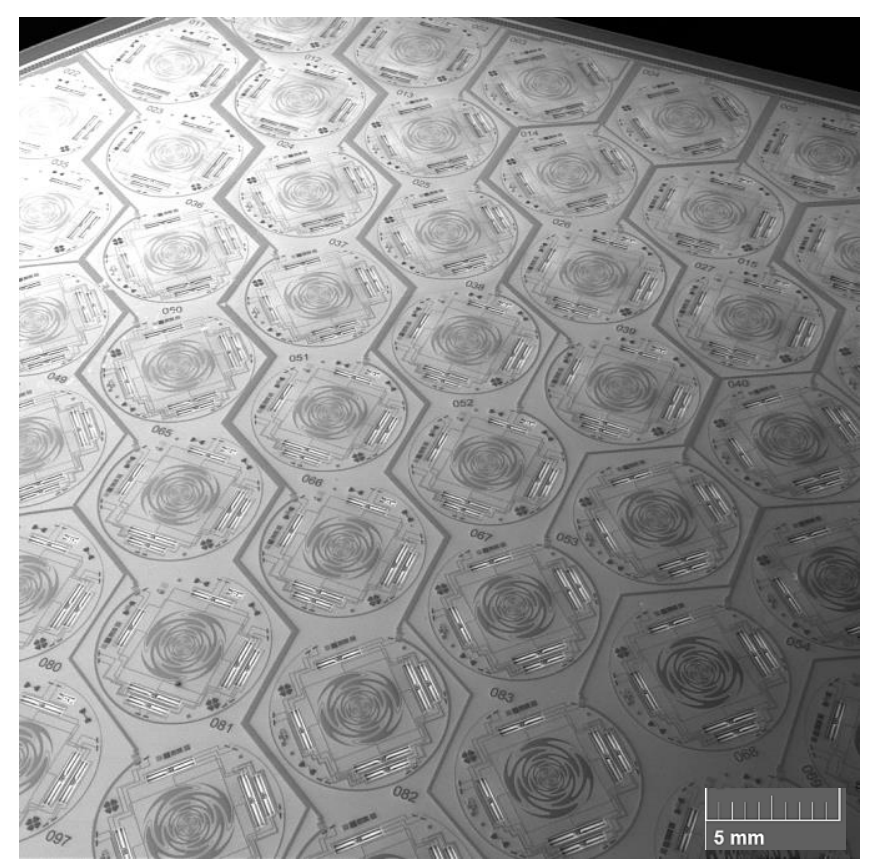

Figure 2. SEM micrograph of a fabricated SPT-3G multichroic pixel array, including the wiring layout. $5 \mu \mathrm{m} \mathrm{Nb}$ traces with a $5 \mu \mathrm{m}$ pitch are routed between pixels to bond-pads located at the edges of the array.

\section{PIXEL DESIGN}

The pixel design builds upon prior development of multichroic dual-polarization bolometric detectors performed at UC Berkeley $[10,11,14,15]$ and TES detector technology at Argonne National Laboratory [16,17]. As shown in Figure 3, each pixel contains a self-complementary, log-periodic, broadband sinuous antenna, sensitive to linear polarizations $[18,19]$.

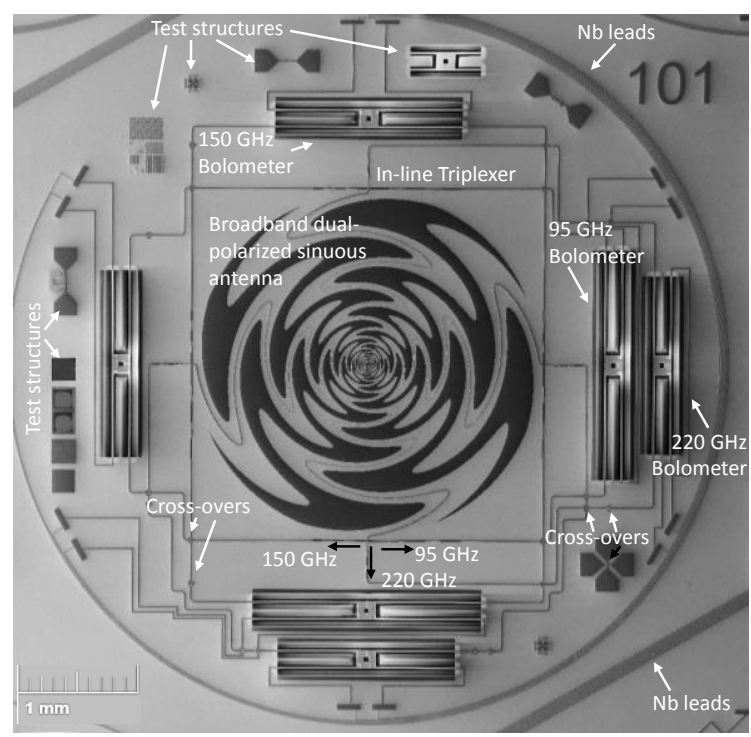

Figure 3. SPT-3G multichroic pixel components. 
The signal detected by the antenna couples to a $\mathrm{Nb}$ microstrip transmission line. In-line three-pole filters are used to define the spectral response before reaching the bolometers. To read out the signal coming from the dual-polarization antenna, cross-over structures separating two different $\mathrm{Nb}$ microstrip transmission lines are fabricated. This allows routing the signals coming from the antenna to their respective detectors. The signal is dissipated as heat by a Ti/Au load resistor and the respective change in temperature is detected by a Ti/Au TES. For each detector, the load resistor and TES thermistor are placed on a thermally-isolated low-stress silicon nitride (LSN) membrane suspended via four narrow legs. A thick Pd layer is deposited on the bolometer island to slow down the thermal response time of the detectors [20,21]. Multiple test structures are placed around the pixel to monitor the different stages of the fabrication process. These test structures include patterns to check for end-points during etching steps, step coverage, alignment test structures, patterns to check for the exposure and development during the lithography steps, and small devices to probe bolometer films. Sonnet and HFSS simulations were used to evaluate the expected behavior of the multichroic pixels and to optimize their polarization sensitivity in the $95 \mathrm{GHz}, 150 \mathrm{GHz}$ and $220 \mathrm{GHz}$ spectral ranges. A summary of the dimensions and design specifications of the SPT-3G detector elements is presented in Table 1.

Table 1. Design parameters of the detector components.

\begin{tabular}{ccc}
\hline Bolometer element & Geometry & Design specifications \\
\hline TES & $30 \mu \mathrm{m} \times 80 \mu \mathrm{m}, \mathrm{t}^{\mathrm{a}}: \mathrm{Ti}(200 \mathrm{~nm}) / \mathrm{Au}(20 \mathrm{~nm})$ & $\mathrm{Tc}^{\mathrm{b}}=510 \mathrm{mK}, \mathrm{Rn}^{\mathrm{c}}=1.2 \Omega$ \\
\hline Load Resistor & 4 squares, $\mathrm{t}: \mathrm{Ti}(40 \mathrm{~nm}) / \mathrm{Au}(5 \mathrm{~nm})$ & $5.5 \Omega / \mathrm{Sq}$. \\
\hline Extra Heat Capacity & $13548 \mu \mathrm{m}^{2}, \mathrm{t}: \mathrm{Ti}(5 \mathrm{~nm}) / \mathrm{Pd}(700 \mathrm{~nm})$ & \\
\hline $95 \mathrm{GHz}$ bolometer length & $1957 \mu \mathrm{m}$ & $\mathrm{P}_{\text {sat }}^{\mathrm{d}}=12.5 \mathrm{pW}\left(2.5 \times \mathrm{PP}_{\text {load }}^{\mathrm{e}}\right)$ \\
\hline $150 \mathrm{GHz}$ bolometer length & $1597 \mu \mathrm{m}$ & $\mathrm{P}_{\text {sat }}=15.0 \mathrm{pW}\left(2.5 \times \mathrm{PP}_{\text {load }}\right)$ \\
\hline $220 \mathrm{GHz}$ bolometer length & $1517 \mu \mathrm{m}$ & $\mathrm{P}_{\text {sat }}=16.8 \mathrm{pW}\left(2.5 \mathrm{xP}_{\text {load }}\right)$ \\
\hline
\end{tabular}

${ }^{\mathrm{a}}$ Film thickness, ${ }^{\mathrm{b}}$ superconducting transition temperature, ${ }^{\mathrm{c}} \mathrm{TES}$ normal resistance, ${ }^{\mathrm{d}}$ expected saturation powers for the detectors, ${ }^{\mathrm{e}}$ expected optical loading power for the detectors.

\section{FABRICATION PROCESS}

\section{Fabrication overview}

Each of the ten detector arrays that will populate the SPT-3G focal plane is fabricated using $150 \mathrm{~mm}$ silicon (100) wafers, $675 \mu \mathrm{m}$ thick, coated with a $1 \mu \mathrm{m}$ layer of LSN deposited by lowpressure chemical vapor deposition (LPCVD). Except for the exposure of the array-level wiring layout, all layers are patterned using stepper-based lithography. An ASML PAS 5000 stepper is used in every case, delivering an overlay tolerance between layers better than $200 \mathrm{~nm}$. A SUSS MA6/BA6 contact aligner is used to pattern the array-level wiring layout. Mask designs and process conditions are selected such that they are compatible with the two i-Line positive photoresists used throughout the process: SPR-955 (Megaposit) and Ultra-i 123 (Electronic Materials). The fabrication work is carried out in the class-100 clean room of the Center for Nanoscale Materials at Argonne National Laboratory. The step-by-step fabrication process flow of the detectors is presented in Figure 4. 

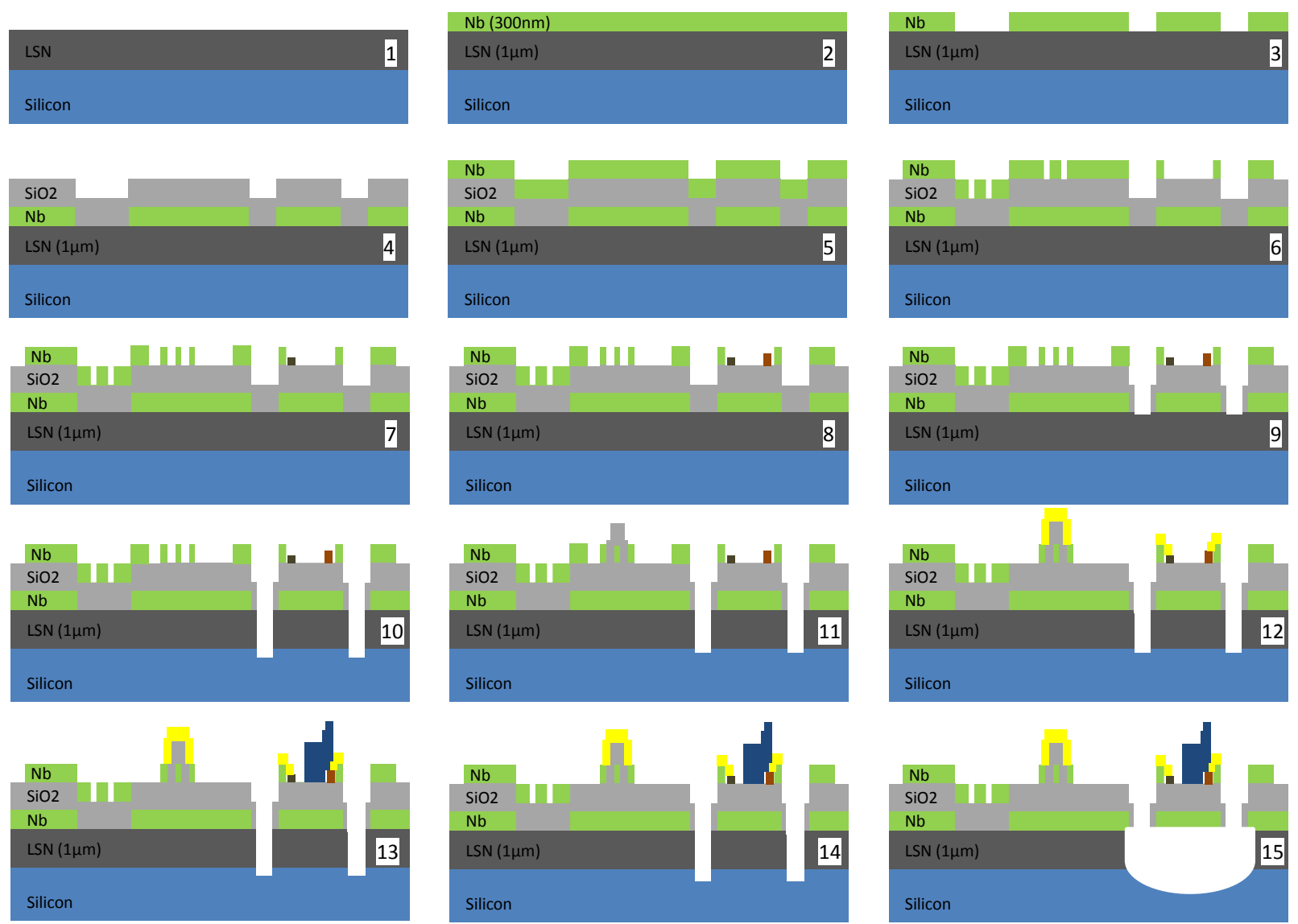

Figure 4: Fabrication process flow showing the thin film layer that make up the multichroic SPT-3G detectors. The numbers accompanying the frames in this figure are associated to the detailed description presented for each step in Table 2.

A total of 12 lithography steps are employed for the fabrication of the SPT-3G detectors. Detector arrays are processed in batches of five wafers. Metallic layers are deposited by DC magnetron sputtering. Dielectric layers are deposited using RF magnetron sputtering and RF reactive sputtering. For better film quality, all depositions are carried out with a base pressure of $5 \times 10^{-8}$ Torr or lower. To minimize the number of pinholes in the dielectric layers, it was found that the deposition vacuum chamber should be opened and cleaned between each batch of five detector arrays. Prior to every deposition in which a metallic layer has been exposed to air, the oxide on the metal surface is removed by argon back-sputtering using a RF bias of $25 \mathrm{~W}$ at 3 mTorr. Scanning electron microscopy (SEM) is used during the process to evaluate the step coverage of the deposited films as well as to visually inspect the different components of the detectors. Energy-dispersive X-ray spectroscopy (EDS) is used to evaluate the elemental composition of the components in the wafer, allowing us to check for possible film contamination or incomplete etching processes. Atomic force microscopy (AFM) is employed to precisely determine the thicknesses of deposited films as well as to image the profile of the detector components after etching or lift-off. Using these techniques allows us to control the quality of the deposited films and to monitor the fabrication process. Details on the process 
parameters used for fabrication are summarized in Table 2. The step number in Table 2 corresponds to the number of each frame shown in Figure 4.

Table 2. Detailed description of the processes used in detector fabrication. The step number in this table identifies the respective frame in the fabrication process flow shown in Figure 4.

\begin{tabular}{|c|c|c|c|c|}
\hline Step & Description & Lithography & Fabrication Process & Thickness \\
\hline 1 & Define a ligment marks on $\mathrm{Si}_{3} \mathrm{~N}_{4}$. Etch & Stepper, PR: SPR 955 & $\begin{array}{l}\text { Oxford ICP/RIE Etch, RF: } 200 \mathrm{~W}, \mathrm{ICP}: 1200 \mathrm{~W}, \mathrm{CHF}_{3}(30 \mathrm{sccm}), \mathrm{Ar} \\
(30 \mathrm{sccm}), 30 \mathrm{mTorr}\end{array}$ & $-140 \mathrm{~nm}$ \\
\hline 2 & Deposition of $\mathrm{Nb}$ ground plane & & $\begin{array}{l}\text { AJA Inc, DC magnetron sputtering, } 250 \mathrm{~W}, 1.3 \mathrm{mT} \text { Torr. Target: } \mathrm{Nb} \\
(0.9995 \%)\end{array}$ & $300 \mathrm{~nm}$ \\
\hline 3 & $\begin{array}{l}\text { Pattern antenna, filters, ground layer and } \\
\text { bolometers slots. Etch }\end{array}$ & Stepper, PR: SPR 955 & $\begin{array}{l}\text { Oxford, ICP/RIE Etch, RF: } 100 \mathrm{~W}, \mathrm{ICP}: 600 \mathrm{~W}, \mathrm{CHF}_{3}(20 \mathrm{sccm}), \mathrm{SF}_{6} \\
(25 \mathrm{sccm}), 12 \mathrm{mT} \text { orr }\end{array}$ & $-300 \mathrm{~nm}$ \\
\hline 4 & Deposition of $\mathrm{SiO}_{\mathrm{x}}$ dielectric & & $\begin{array}{l}\text { AJA Inc, RF magnetron reactive sputtering, } 250 \mathrm{~W}, 1.3 \mathrm{mTorr}, 250^{\circ} \mathrm{C} \text {, } \\
37 \mathrm{~W} \text { RF bias, } \operatorname{Ar}(27.6 \mathrm{sccm}), \mathrm{O}_{2}(2.4 \mathrm{sccm}) \text {. Target: } \mathrm{Si}(0.9995 \%)\end{array}$ & $500 \mathrm{~nm}$ \\
\hline 5 & Deposit $\mathrm{Nb}$ for microstrip and leads & & $\begin{array}{l}\text { AJA Inc, DC magnetron sputtering, } 250 \mathrm{~W}, 1.3 \mathrm{~m} \text { Torr. Target: } \mathrm{Nb} \\
(0.9995 \%)\end{array}$ & $300 \mathrm{~nm}$ \\
\hline 6 & $\begin{array}{l}\text { Pattern microstrip, leads, filter's top layer } \\
\text { and cross-overs bottom layer }\end{array}$ & $\begin{array}{l}\text { Stepper + Optical, PR: } \\
\text { SPR } 955\end{array}$ & $\begin{array}{l}\text { Oxford, ICP/RIE Etch, RF: } 100 \mathrm{~W}, \mathrm{ICP}: 600 \mathrm{~W}, \mathrm{CHF}_{3}(20 \mathrm{sccm}), \mathrm{SF}_{6} \\
(25 \mathrm{sccm}), 12 \text { mTorr }\end{array}$ & $-300 \mathrm{~nm}$ \\
\hline 7 & Pattern Ti/Au TES. Lift-off & $\begin{array}{l}\text { Stepper, PR: LOR-3A / } \\
\text { Ultra-i } 123\end{array}$ & $\begin{array}{l}\text { AJA Inc, DC magnetron sputtering, Target: Ti }(0.9995), 260 \mathrm{~W} \text {, } \\
\text { 1.6mTorr / Target: } \mathrm{Au}(0.9999 \%), 75 \mathrm{~W}, 3.2 \mathrm{mTorr}\end{array}$ & $\begin{array}{l}200 \mathrm{~nm}(\mathrm{Ti}) / \\
20 \mathrm{~nm}(\mathrm{Au})\end{array}$ \\
\hline 8 & Pattern Ti/Au Resistor. Lift-off & $\begin{array}{l}\text { Stepper, PR: LOR-3A / } \\
\text { Ultra-i } 123\end{array}$ & $\begin{array}{l}\text { AJA Inc, DC magnetron sputtering, Target: Ti }(0.9995 \%), 260 \mathrm{~W} \text {, } \\
1.6 \mathrm{mT} \text { Torr / Target: } \mathrm{Au}(0.9999 \%), 75 \mathrm{~W}, 3.2 \mathrm{mTorr}\end{array}$ & $\begin{array}{l}40 \mathrm{~nm}(\mathrm{Ti}) / 5 \mathrm{~nm} \\
(\mathrm{Au})\end{array}$ \\
\hline 9 & $\begin{array}{l}\text { Remove } \mathrm{SiO}_{\mathrm{x}} \text { from bolometers legs and } \\
\text { open } \mathrm{Nb} \text { ground plane. Etch }\end{array}$ & Stepper, PR: SPR 955 & $\begin{array}{l}\text { Oxford, ICP/RIE Etch, RF: } 200 \mathrm{~W}, \mathrm{ICP}: 1200 \mathrm{~W}, \mathrm{CHF} 3(30 \mathrm{sccm}), \mathrm{Ar} \\
(30 \mathrm{sccm}), 30 \mathrm{mT} \text { orr }\end{array}$ & $-500 \mathrm{~nm}$ \\
\hline 10 & Remove LSN from bolometers legs. Etch & Stepper, PR: SPR 955 & $\begin{array}{l}\text { Oxford, ICP/RIE Etch, RF: } 200 \mathrm{~W}, \mathrm{ICP}: 1200 \mathrm{~W}, \mathrm{CHF} 3(30 \mathrm{sccm}), \mathrm{Ar} \\
(30 \mathrm{sccm}), 30 \mathrm{mT} \text { orr }\end{array}$ & $-1000 \mathrm{~nm}$ \\
\hline 11 & $\begin{array}{l}\text { Pattern } \mathrm{SiO}_{2} \text { dielectric spacer for cross- } \\
\text { overs. Lift-off }\end{array}$ & $\begin{array}{l}\text { Stepper, PR: LOR-3A / } \\
\text { Ultra-i } 123\end{array}$ & $\begin{array}{l}\text { AJA Inc, RF magnetron sputtering, } 100 \mathrm{~W}, 3.0 \mathrm{mT} \text { orr. } 25 \mathrm{~W} \text { RF bias. } \\
\text { Target: } \mathrm{SiO}_{2}(0.9995 \%)\end{array}$ & $350 \mathrm{~nm}$ \\
\hline 12 & $\begin{array}{l}\text { Complete cross-overs with } \mathrm{Nb} \text { top layer } \\
\text { (bridges). Lift-off }\end{array}$ & $\begin{array}{l}\text { Stepper, PR: LOR-3A/ } \\
\text { Ultra-i } 123\end{array}$ & $\begin{array}{l}\text { AJA Inc, DC magnetron sputtering, } 250 \mathrm{~W}, 1.3 \mathrm{mT} \text { Torr. Target: } \mathrm{Nb} \\
(0.9995 \%)\end{array}$ & $400 \mathrm{~nm}$ \\
\hline 13 & $\begin{array}{l}\text { Deposit Pd layer for extra-heat capacity. } \\
\text { Lift-off }\end{array}$ & $\begin{array}{l}\text { Stepper, PR: LOR-3A / } \\
\text { Ultra-i } 123\end{array}$ & $\begin{array}{l}\text { AJA Inc, DC magnetron sputtering, Target: Ti }(0.9995 \%), 260 \mathrm{~W} \text {, } \\
1.6 \mathrm{mT} \text { orr / Target: } \mathrm{Pd}(0.9995 \%), 75 \mathrm{~W}, 3.0 \mathrm{mTorr}\end{array}$ & $\begin{array}{l}5 \mathrm{~nm}(\mathrm{Ti}) / \\
700 \mathrm{~nm}(\mathrm{Pd})\end{array}$ \\
\hline 14 & Dice wafer & & & \\
\hline 15 & $\begin{array}{l}\text { Remove Si to define the detectors weak } \\
\text { link. Bolometers relase. Etch }\end{array}$ & Stepper, PR: SPR 955 & $\mathrm{XeF}_{2} \mathrm{Si}$ etch. 3Torr, Cycle duration: 30sec, 165 cycles & \\
\hline 16 & Remove photoresist SPR 955. Etch & & Oxford, ICP/RIE Etch, RF: 80W, ICP: $800 \mathrm{~W}, \mathrm{O}_{2}(50 \mathrm{sccm}), 20 \mathrm{mTorr}$ & $-2000 \mathrm{~nm}$ \\
\hline
\end{tabular}

\section{Sinuous antenna and microstrip transmission line}

The SPT-3G sinuous antenna is a log-periodic antenna with four arms, in which two opposite arms couple to a single linear polarization [18]. Due to the self-complementary architecture of the antenna, it has nearly frequency-independent input impedance [19,21-23]. However, for this type of antenna, the plane of polarization changes periodically as a function of frequency, with an approximate amplitude of 5 degrees [19]. Therefore, to remove the bias from this systematic behavior, the array is made up of pairs of left-handed (A) and right-handed (B) pixels, in which the antennas are mirror images of each other, as shown in Figure 5. In order to fully sample the polarization signal on the sky, the array consists of pairs of pixels rotated by 45 degrees, which measure Stokes Q and U parameters of incoming sky radiation [23]. 


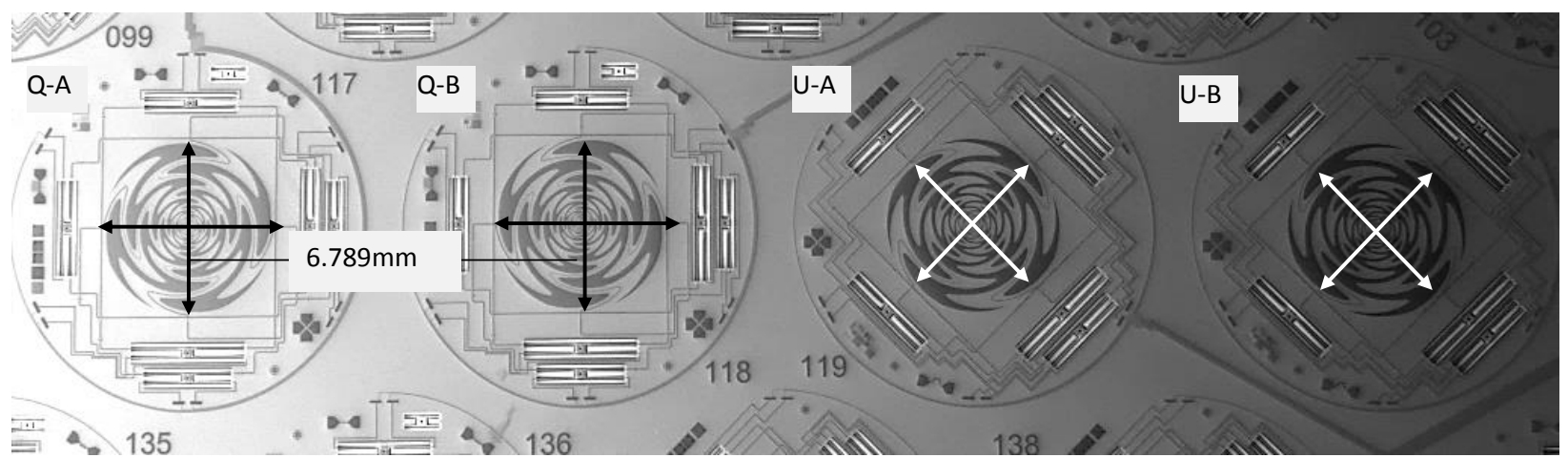

Figure 5. SEM micrograph showing the disposition of pairs of $Q$ and $U$ pixels within the array. Antennas in pixels $\mathrm{A}$ and $\mathrm{B}$ are mirrored with respect to each other.

In the initial fabrication stages, the antenna is etched away from the $300 \mathrm{~nm}$ of $\mathrm{Nb}$ ground plane. The end-point detector of the inductively coupled plasma reactive ion etching (ICP-RIE) system used in this step allows us to accurately stop the etching process and avoid substantial over-etch of the ground plane. The $\mathrm{Nb}$ etching process (see Table 2) was adjusted to obtain straight walls, as well as a uniform etching rate across the $135 \mathrm{~mm}$ in diameter circular area occupied on the wafer by the detectors. The continuous metallic arms of the antenna are used as the ground plane for the microstrip transmission line, bringing the signal from the antenna to the bolometers. This microstrip line consists of $300 \mathrm{~nm}$ Nb superconducting ground plane, $500 \mathrm{~nm}$ $\mathrm{SiO}_{\mathrm{x}}$ (deposited by RF magnetron reactive sputtering), and a $300 \mathrm{~nm} \mathrm{Nb}$ microstrip layer. To achieve a more conformal dielectric film, the wafer is heated to $250^{\circ} \mathrm{C}$ and $37 \mathrm{~W} \mathrm{RF}$ bias is applied during deposition of the dielectric layer [24]. Images of the microstrip and the center of the antenna are shown in Figure 6.
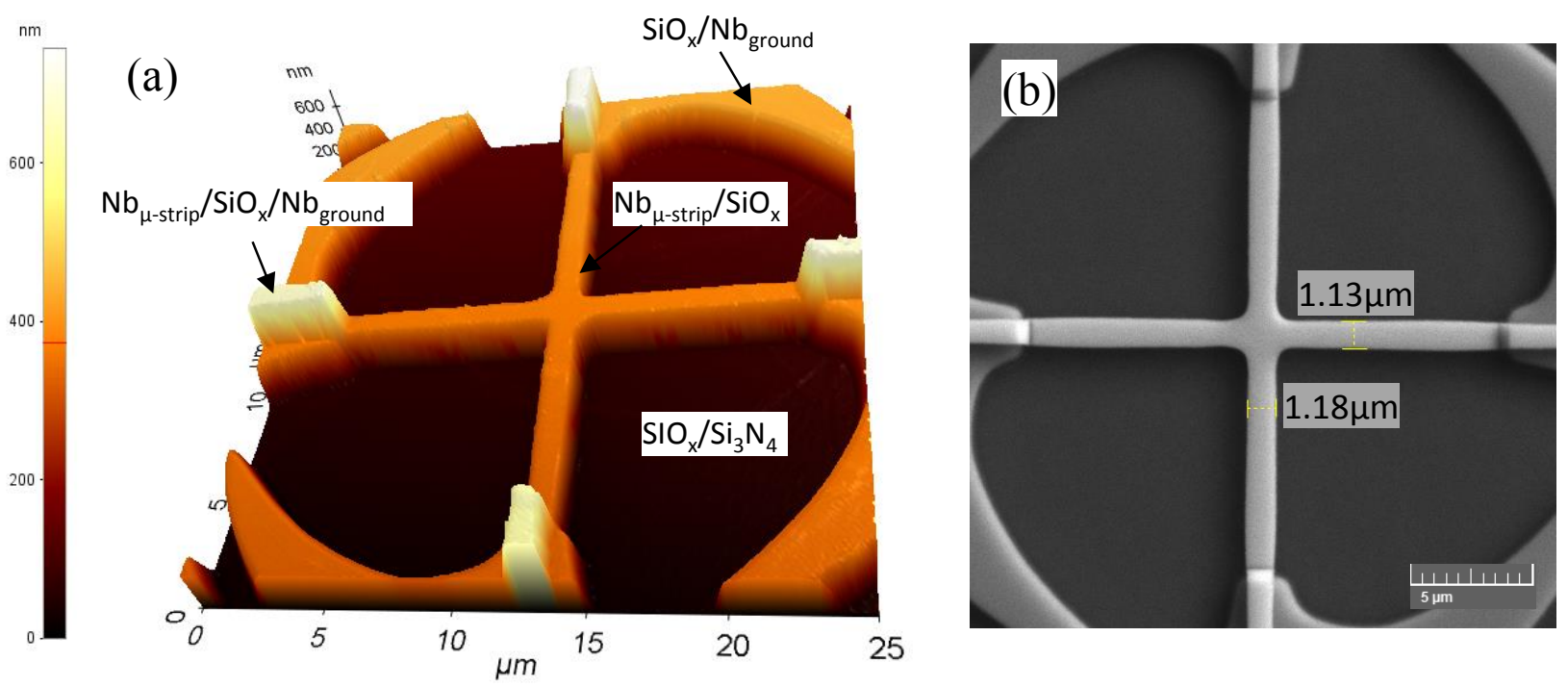

Figure 6. (a) AFM image of the center of the antenna. The narrow lines correspond to the $\mathrm{Nb}$ microstrip line that is deposited on the metallic arms of the sinuous antenna. (b) SEM image showing the microstrip dimensions in the center of the antenna. Target width: $1.2 \mu \mathrm{m}$. 
At the center of the antenna, a $1.2 \mu \mathrm{m}$ wide microstrip is required to match the impedance of the antenna. As shown in Figure 6 (b), variations in the microstrip width below $100 \mathrm{~nm}$ were observed during fabrication, for which reflection is expected to be small [23]. As the microstrip moves away from the center of the antenna, its width gradually increases to $10 \mu \mathrm{m}$. A gradual taper from a width of $1.2 \mu \mathrm{m}$ to a width of $10 \mu \mathrm{m}$ transforms the microstrip impedance from $50 \Omega$ to $10 \Omega$.

To minimize changes in the transmission line phase velocity, the thickness of the microstrip was chosen to be at least five times larger than the typical London penetration depth of $\mathrm{Nb}[23$, 25]. To estimate the dielectric loss tangent $(\tan \delta)$ for the microstrip and to optimize the dielectric deposition parameters, $\mathrm{Nb}$ microstrip line resonators were fabricated and tested at frequencies between $5 \mathrm{GHz}$ and $10 \mathrm{GHz}$ [24]. From these resonators, a dielectric loss tangent of $1.7 \times 10^{-3}$ was measured [24]. Considering the microstrip length $(\sim 16 \mathrm{~mm})$, the estimated attenuation of the input signal power due to loss in the dielectric is on the order of $7 \%, 10 \%$ and $13 \%$ for the 95 $\mathrm{GHz}, 150 \mathrm{GHz}$ and $220 \mathrm{GHz}$ detectors, respectively. Additional input signal power attenuation on the order of $10 \%$ is expected for each frequency due to losses in the filters.

\section{Lumped triplexer}

Three-pole quasi-lumped element triplexer filters split the broadband signal and define the center frequency and bandwidth of our detectors. A demonstration of the working characteristics of these triplexer filters [15], as well as details on the design and optimization of these filters can be found elsewhere [22,23,26,27]. In general, these filters are made up of short high impedance sections acting as inductors and parallel plate capacitors and are fabricated using the same layers as the microstrip transmission line [14,15,27]. The expected detector band-passes are plotted in Figure 7 together with atmospheric transmission expected for the typical values of precipitable water vapor (PWV) found at the South Pole. The bands are selected such that atmospheric absorption in the millimeter range is avoided.

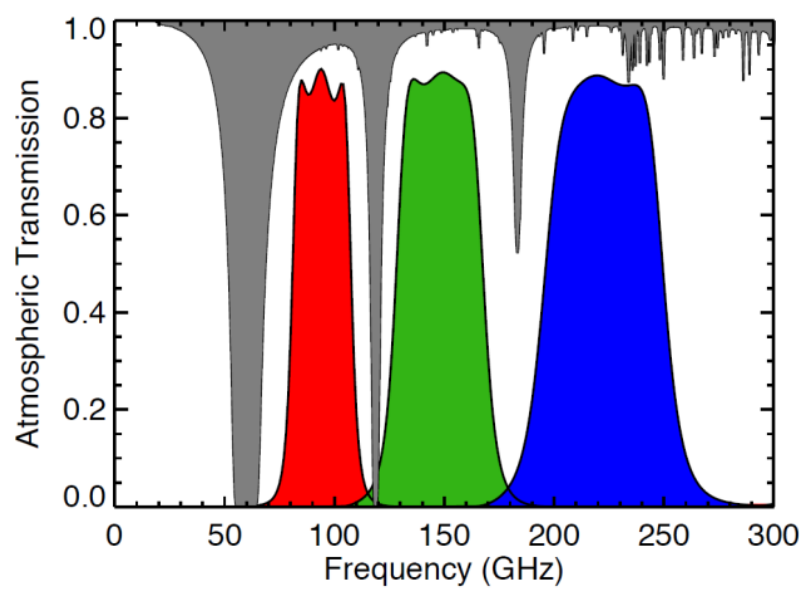

Figure 7. Expected filter band-passes in comparison with atmospheric transmission (grey area) for the typical values of PWV=0.25 $\mathrm{mm}$ found at the South Pole. 
A 2.5 dimension electro-magnetic simulator (Sonnet) was used to evaluate the robustness of the triplexer design. Results indicated that variations on the order of $+/-200 \mathrm{~nm}$ in the size of the filter features will have negligible effect on the resulting band-passes. Variations on the dielectric thickness will affect the filter's performce as it will modify the capacitance of their parallel plate capacitor sections. Sonnet simulation results indicate that changes of up to $8 \%$ in the dielectric thickness will shift the band-passes by less than $5 \mathrm{GHz}$. A reduction in thickness will result in a shift to lower frequencies, while a thicker dielectric will lead to a shift to higher frequencies (see Figure 8). To minimize the effect of the dielectric thickness, the microstrip line resonators fabricated to estimate the losses in the microstrip transmission line [24] are also used as test devices to monitor $\mathrm{Nb}$ and $\mathrm{SiO}_{\mathrm{x}}$ thickness variations between batches of detector arrays. Consequently, the variation on the thickness of the deposited $\mathrm{SiO}_{\mathrm{x}}$ is kept below $+/-40 \mathrm{~nm}$.

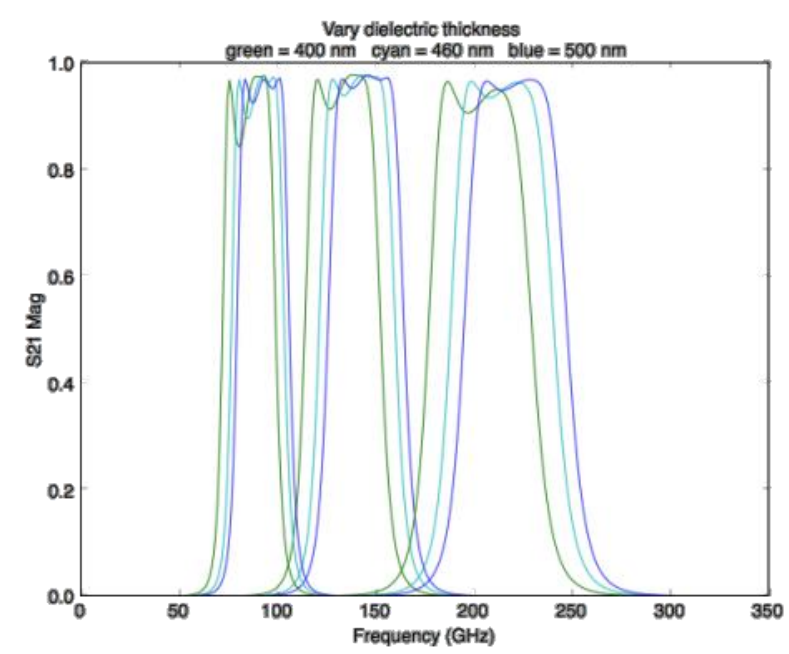

Figure 8. Effect of reducing the dielectric thickness on the filter band-passes.

\section{Transition edge sensor}

A Ti $(200 \mathrm{~nm}) / \mathrm{Au}(20 \mathrm{~nm})$ bilayer is used as the TES material. The TES layer is patterned by the stepper using a $300 \mathrm{~nm}$ lift-off resist (LOR-3A) in combination with the positive photoresist Ultra-i 123. The use of LOR-3A yields smoother edges of the thin film and simplifies the lift-off process over the $150 \mathrm{~mm}$ substrate. The Ti and Au targets are located in the same deposition chamber. This allows us to perform the deposition of the bilayer without breaking vacuum, thus minimizing the formation of oxide at the metal interface. The thin gold layer is deposited to prevent the oxidation of the Ti surface overtime. The measured transition temperature of one equivalent square sample of a Ti/Au TES is found to be around $515 \mathrm{mK}$ to $540 \mathrm{mK}$, and has an approximate normal resistance of $0.55 \Omega / \mathrm{Sq}$., as shown in Figure 9 . A similar transition temperature has been reported for high purity samples of $\mathrm{Ti}$ [28].

The transition temperature of the deposited $\mathrm{Ti} / \mathrm{Au}$ films has been found to be affected by the deposition base pressure and the processing conditions after patterning. In particular, it has been observed that heating the wafers at temperatures higher than $150^{\circ} \mathrm{C}$ can lead to a decrease in the TES transition temperature $\left(T_{c}\right)$ in the order of 10 to $20 \%$. Consequently, the TES films are deposited at a base pressure below $2 \times 10^{-8}$ Torr, and temperatures above $110^{\circ} \mathrm{C}$ are avoided in the fabrication after the TES film is deposited. 


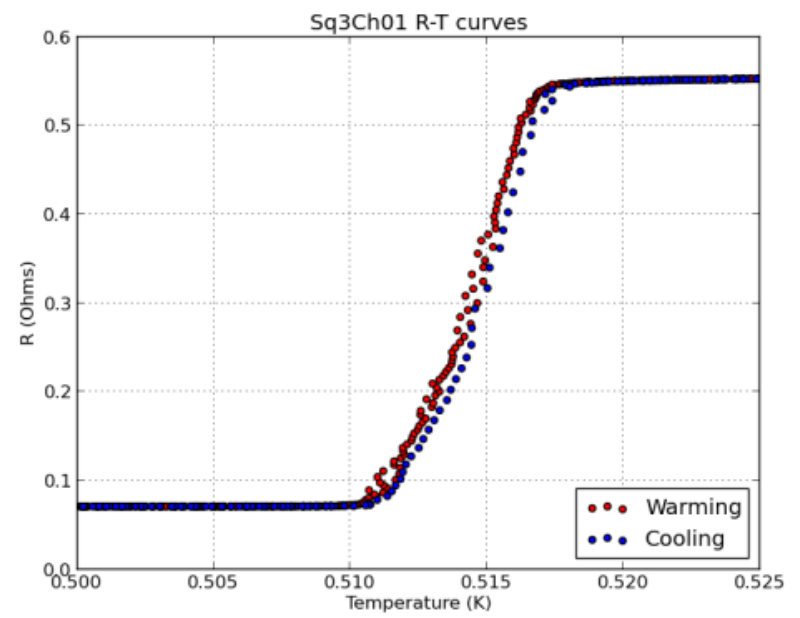

Figure 9. Superconducting-to-normal resistive transition profile of 1 square $\mathrm{Ti}(200 \mathrm{~nm}) / \mathrm{Au}$ (20nm) bilayer TES.

Recent work has shown that the superconducting proximity in the lateral dimension can produce modifications of the resistive transition profile due to the denominated lateral proximity effect $[29,30,31]$. This effect modifies the resistive transition profile, helping to simultaneously tailor the superconducting, electrical, and thermal properties of the bolometer. Effects of the lateral proximity effect include changing $\mathrm{R}(\mathrm{T})$ at temperatures above the superconducting transition temperature $\left(T_{c}\right)$ allowing for operation under high loading conditions, a non-zero resistance below $T_{c}$ preventing the detector from latching, and a soft transition that keeps the loop gain low providing for more stable TES operation [17, 21, 29-31]. This effect has been shown previously in bolometer designs implemented in the $95 \mathrm{GHz}$ SPTpol detectors [16]. In addition to the Ti/Au bilayer, our TES may be taking advantage of this lateral proximity effect by placing a normal metal such as Pd in series with the TES material. As a result, this effect may be broadening our film transitions, helping to prevent thermal instability deep in the transition [17]. Additional test devices are being fabricated to investigate in detail the influence of this lateral proximity effect on the resistive transition profile of our films.

\section{Ti/Au load resistor}

A Ti $(40 \mathrm{~nm}) / \mathrm{Au}(5 \mathrm{~nm})$ bilayer termination resistor equivalent to 4 squares is deposited using a similar procedure to the one employed for the TES. Because the critical temperature for this Ti/Au resistor is $\sim 0.5 \mathrm{~K}$ or lower, photons with frequencies larger than $36 \mathrm{GHz}$ will break Cooper pairs. As a consequence, the load resistor operates in its normal state at the target frequencies of our detectors. The resistors have a square resistance of $5.5 \Omega / \mathrm{Sq}$. at room temperature and the 4 squares geometry minimizes reflection from the incoming $10 \Omega$ microstrip lines.

\section{Extra heat capacity}

To add heat capacity to the TES island and obtain a time constant suitable for stable readout, a $700 \mathrm{~nm}$ layer of Pd is deposited on the bolometer island using lift-off. To ensure good thermal 
coupling between this Pd layer and the TES, the detectors are designed with a $5 \mu \mathrm{m} \mathrm{TiAu-Pd}$ overlap, and additional $5 \mu \mathrm{m}$ TiAu-Nb-Pd overlap on each side of the TES (Figure 10).

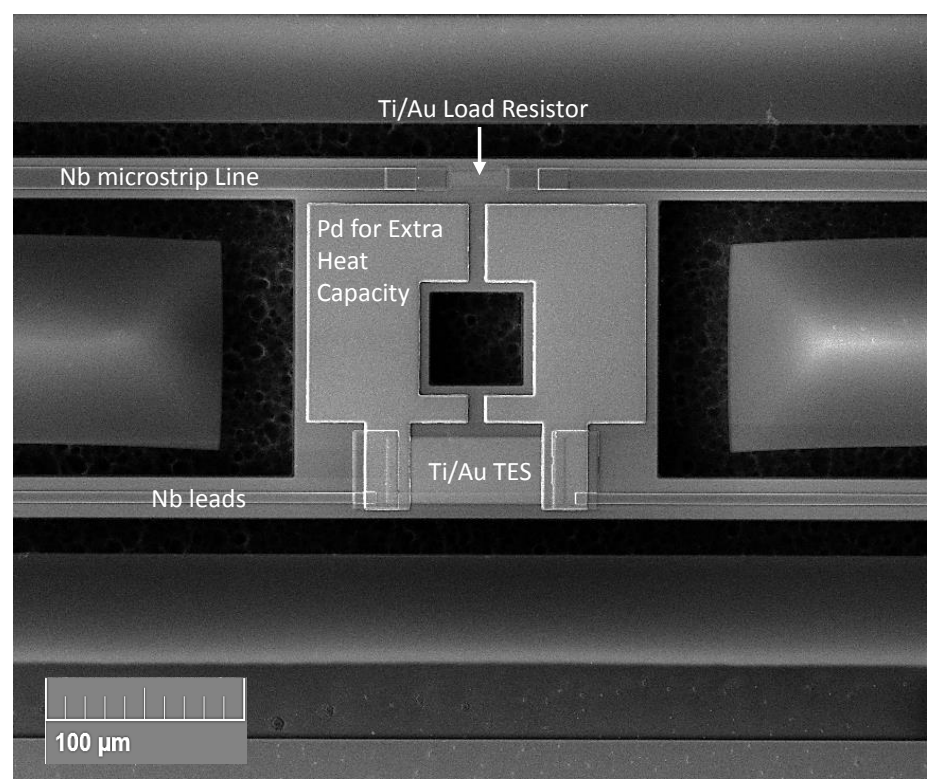

Figure 10. SEM micrograph of the released bolometer island.

Cross-over structures

To read out the signal coming from the four sides of the dual-polarization antenna, cross-over structures were developed. These cross-over structures are composed of a $350 \mathrm{~nm}$ layer of $\mathrm{SiO}_{2}$ separating the $300 \mathrm{~nm} \mathrm{Nb}$ microstrip layer and the $400 \mathrm{~nm} \mathrm{Nb}$ cross-over layer, as shown in Figure 11. In total, each pixel has 21 cross-over structures. The cross-over of the microstrip and top $\mathrm{Nb}$ layers is limited to $4 \mu \mathrm{m} \times 4 \mu \mathrm{m}$ to reduce the capacitive coupling between the two orthogonal polarizations.

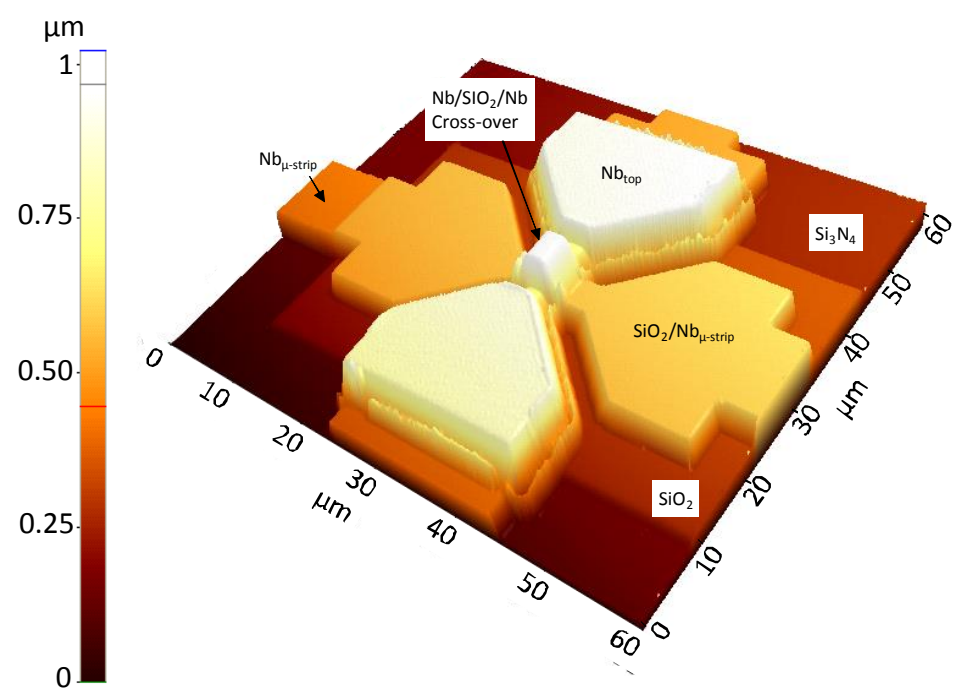

Figure 11. AFM image of $\mathrm{Nb} / \mathrm{SiO}_{2} / \mathrm{Nb}$ cross-over structure. 
To simplify the fabrication process and to avoid contamination of the other elements in the pixels, both the $\mathrm{SiO}_{2}$ and top $\mathrm{Nb}$ layer are patterned by lift-off. In both cases, a bilayer resist composed of LOR-3A and Ultra i-123 is used. To pattern the $\mathrm{SiO}_{2}$ by lift-off, this film is deposited at room temperature from a $\mathrm{SiO}_{2}$ target, using a magnetron sputtering system. The chamber pressure is kept at $3 \mathrm{mTorr}$, and a $25 \mathrm{~W}$ RF bias is applied to improve the step coverage of the resulting dielectric film. SEM micrographs showing a cross-sectional view of the dielectric obtained by lift-off with and without RF bias are shown in Figure 12.

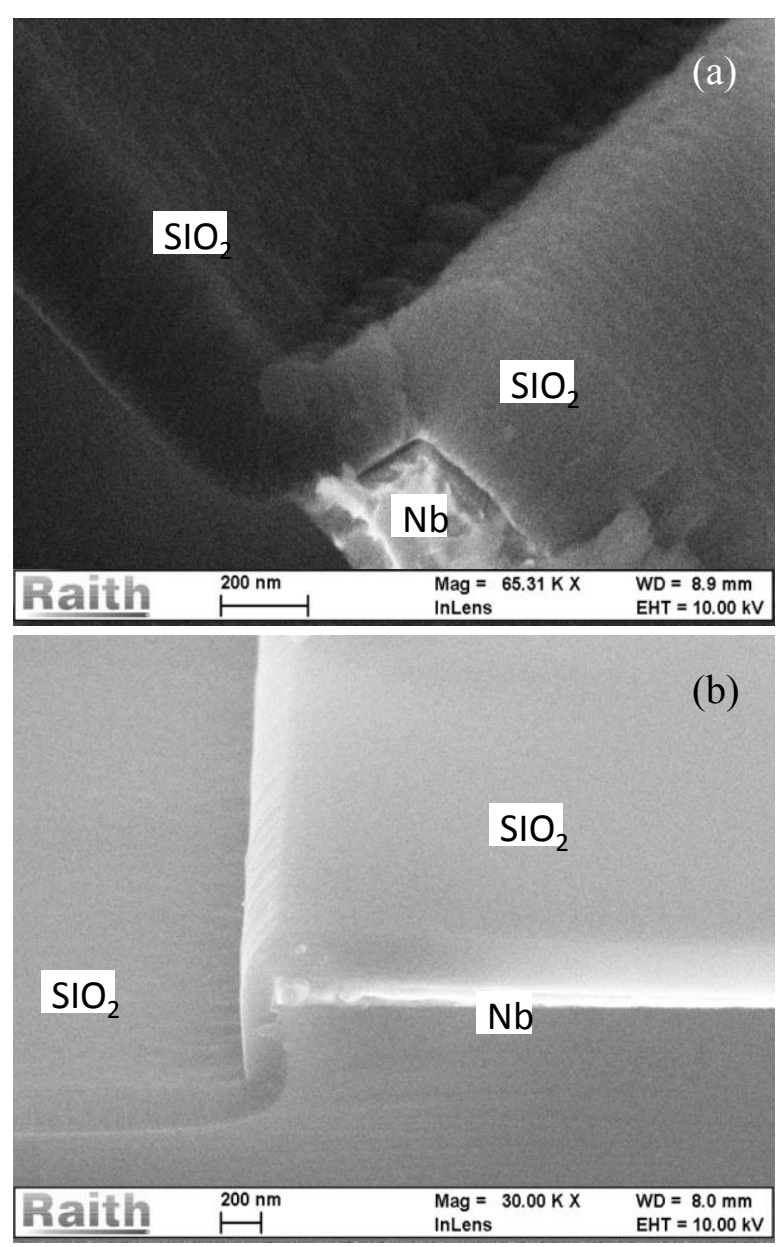

Figure 12. SEM micrographs of the room temperature $\mathrm{SiO}_{2}$ dielectric deposited for the crossover structures. (a) No DC bias applied during deposition. (b) 25W RF Bias applied during deposition. Smoother step coverage for the $\mathrm{SiO}_{2}$ layer over the $\mathrm{Nb}$ is obtained when $\mathrm{RF}$ bias is applied.

\section{Thermal link}

To define the thermal link of the detectors, the bolometers are released by etching the $\mathrm{Si}$ substrate under the island and the legs supporting it. Initially, slots are defined by etching trenches in the $\mathrm{Nb}$ ground plane. HFSS simulations showed that the legs in the bolometers can 
act as slotted antennas, leading to unintended signal pick up. To mitigate this effect, the detectors are fabricated with $100 \mu \mathrm{m}$ wide outer slots and the ground plane between legs is also removed. According to simulation results, a reduction on the order of $80 \%$ in the unintended signal pick up is expected for this geometry when compared to bolometers with $20 \mu \mathrm{m}$ wide outer slots and ground plane between the legs.

Patterning and etching of the slots in the $\mathrm{Nb}$ ground plane for the bolometers is carried out in the same fabrication step as the antenna, corresponding to step 3 in Table 2. The next processes in defining the bolometers weak link include etching $500 \mathrm{~nm} \mathrm{SiO}$ and $1 \mu \mathrm{m} \mathrm{LSN}$ to make narrow trenches around the legs and in the center of the island. Since the etching recipe used to remove $\mathrm{SiO}_{2}$ and LSN also strips the photoresist, these two films are etched using two different lithography steps.

After completing the deposition of Pd for extra heat capacity, and before releasing the LSN membranes, the $150 \mathrm{~mm}$ substrates are cut into their final hexagonal shape using a Thermocarbon dicing saw. The photoresist used to pattern the trenches where the Si substrate will be removed protects the wafer from dust particles generated during dicing. Isotropic $\mathrm{XeF}_{2}$ chemical dry etching is used to remove the Si beneath the released thermistor island. Si dicing debris on the surface of the resist is also removed in this step. The geometry of the LSN legs determines the strength of the thermal link $(G)$ for the bolometer in each band. Table 1 shows target saturation powers and corresponding lengths extrapolated from dark tests of prototype bolometers.

To complete the fabrication process, $\mathrm{O}_{2}$ plasma is used to remove the resist protecting the elements in the array during $\mathrm{XeF}_{2}$ etching. Figures 13 (a) and (b) show optical micrographs of two of our bolometers after $\mathrm{XeF}_{2}$ etching. Figure 13 (c) shows an SEM image of the SPT-3G multichroic pixels after fabrication is completed.
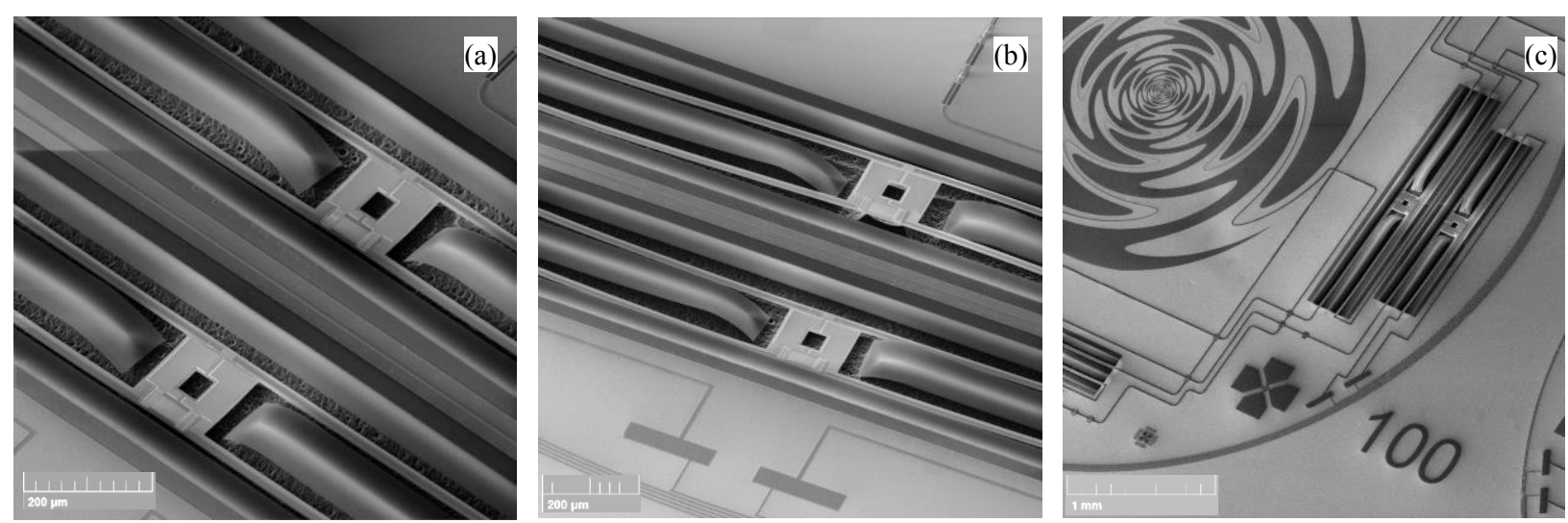

Figure 13. (a) and (b) SEM micrographs of two released bolometers. (c) SEM micrograph of a SPT-3G multichroic pixel after fabrication is completed. 


\section{PRELIMINARY RESULTS}

\section{Optical characterization}

Full characterization of the fabricated array of detectors is still ongoing. Once completed, a manuscript presenting the results obtained from this characterization will be submitted for publication. Nonetheless, preliminary results obtained from prototype detector samples are presented in this section. Figure 14 shows the band-pass and beam map obtained for a $95 \mathrm{GHz}$ detector of a small array of 2 by 3 pixels fabricated according to the procedure described in this paper. The measured band-pass is compared to Sonnet simulation results obtained for the threepole quasi-lumped element triplexer filters. A good match is observed for Sonnet simulations run for a $460 \mathrm{~nm}$ thick dielectric film, which corresponds to the measured dielectric thickness in the measured sample. This dielectric thickness is monitored regularly to meet the target of $500 \mathrm{~nm}$ during the fabrication of the full array detector wafers.

The beam map shown in Figure 14 (b) was taken with 2.5 seconds integration time per step for a detector in the $\mathrm{x}$-polarization. As seen, this beam map is slightly elongated in the $\mathrm{x}$-direction.

(a)
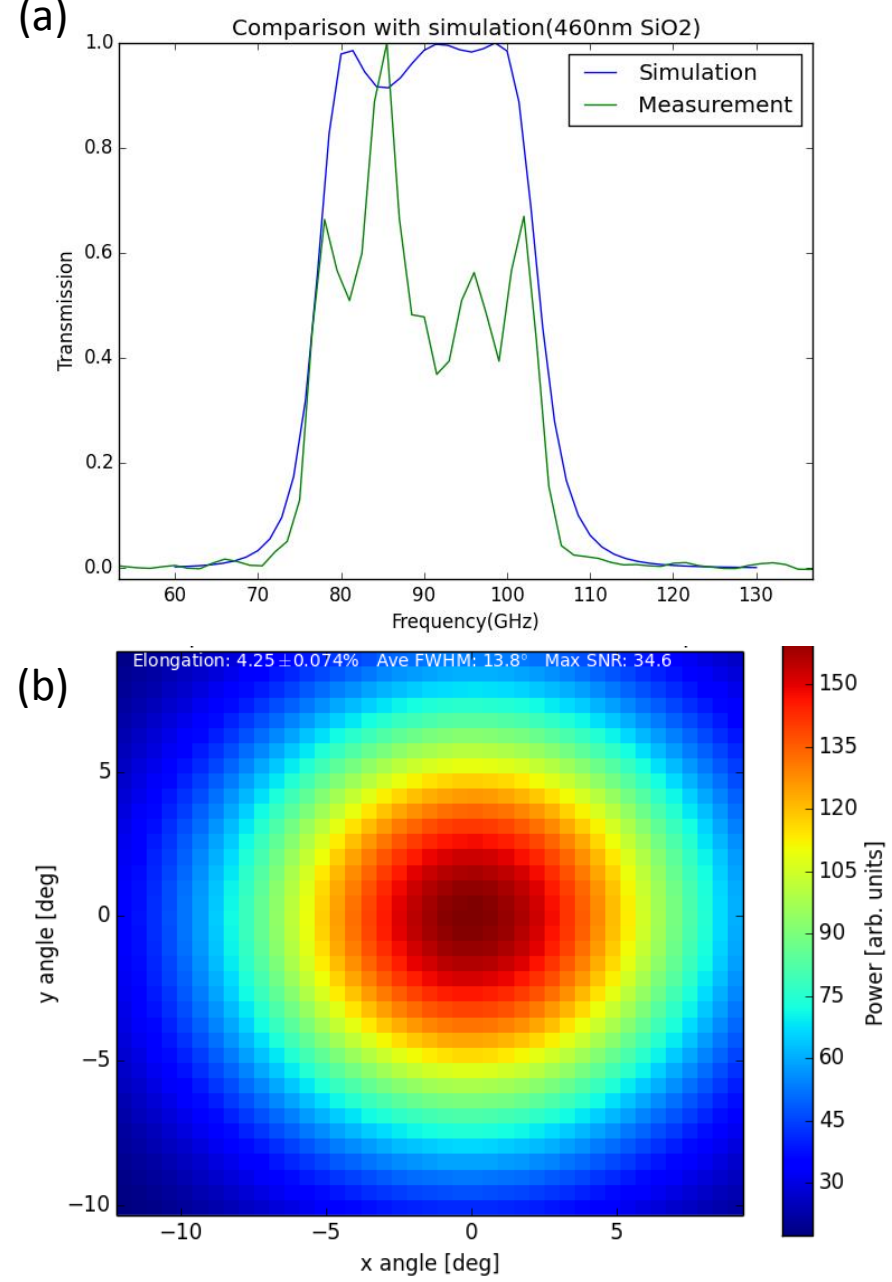

Figure 14. (a) Measured band-pass for a $95 \mathrm{GHz}$ detector compared to Sonnet simulations results a $95 \mathrm{GHz}$ filter fabricated with a $460 \mathrm{~nm}$ thick dielectric film. (b) Beam map obtained for a $95 \mathrm{GHz}$ detector. 


\section{Transition edge sensors characterization}

Figures 15 (a) shows a typical $\mathrm{R}(\mathrm{T})$ curve obtained from our films after fabrication is completed. These curves were taken from small arrays of detectors fabricated using the procedure presented in this paper. The transition temperature of the Ti/Au TES film is generally found to be around $515-540 \mathrm{mK}$. The shape of the transition is affected by the presence of Pd and $\mathrm{Nb}$ at the edges of the TES film, as shown in Figure 10 [21]. Typical values of $\alpha$ for these films are between 200 and 250, as shown in Figure 15 (b). Where $\alpha$ is defined as: $\alpha=$ $\left(\partial \ln \left(R_{-} T E S\right)\right) /(\partial \ln (T))$.
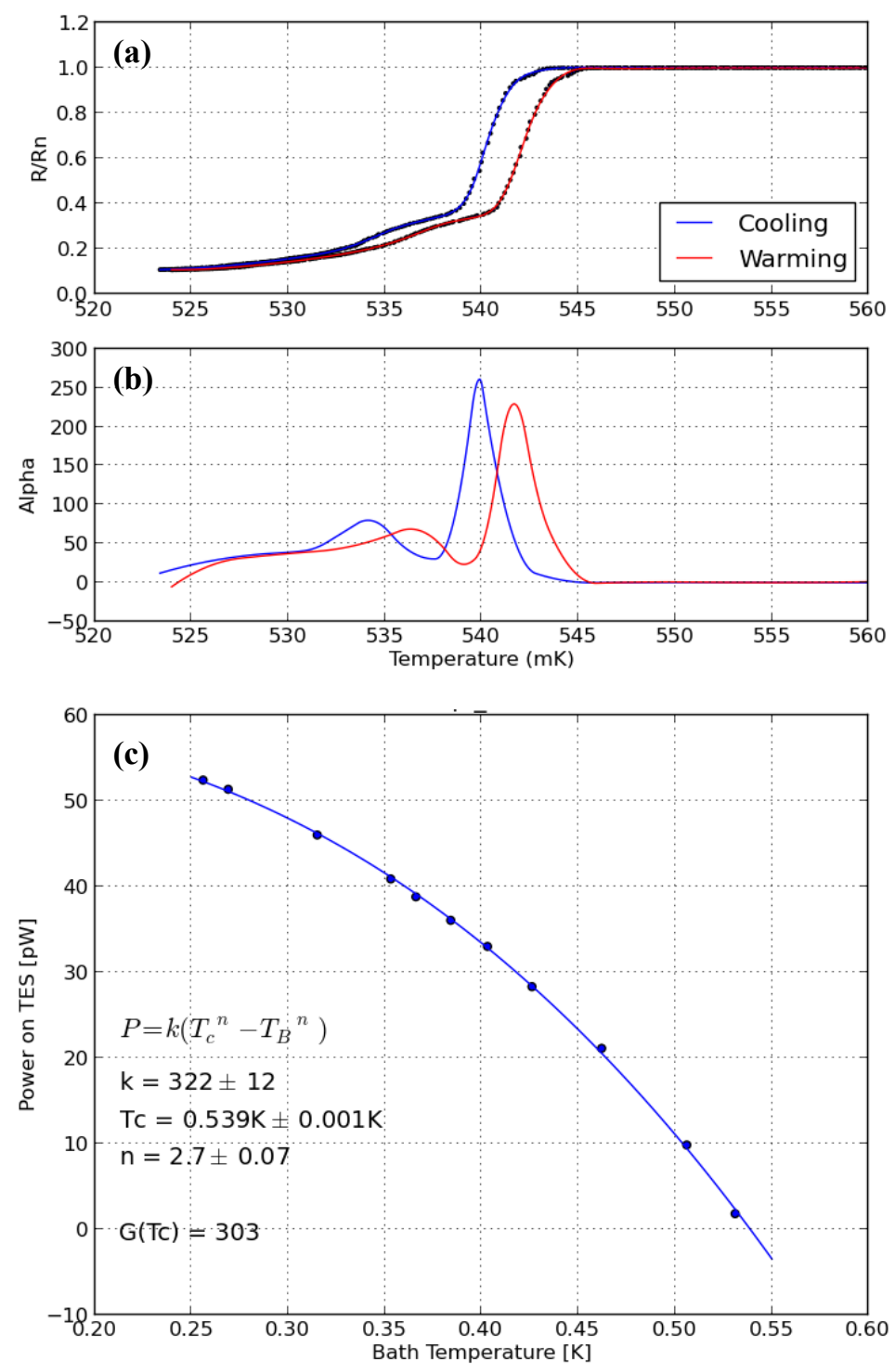

Figure 15. (a) $\mathrm{R}(\mathrm{T})$ curve for the $\mathrm{Ti}(200 \mathrm{~nm}) / \mathrm{Au}(20 \mathrm{~nm}) \mathrm{TES}$ films after fabrication of the detectors is completed, (b) calculation of the logarithmic derivative of resistance with temperature for the TES shown in Figure 15 (a). (c) Calculation of the thermal conductance of one of the fabricated detectors. 
The evaluation of the conductance $\mathrm{G}\left(T_{c}\right)$ is shown in Figure 15 (c). Typical values of $300+/-20$ $\mathrm{pW}$ were obtained for the detectors tested. The exponent $\mathrm{n}$ in Figure 15 (c) indicates the thermal carrier in the weak link; $n=1$ indicates that electrons are the main thermal carriers and $n=3$ corresponds to phonons. In general, the fabricated detectors have $\mathrm{n} \sim 2.7$ to 3.0 , indicating that phonons are the main thermal carriers. Results shown in Figure 15 (c) are from detectors with legs $20-30 \%$ shorter than the current full array design. Consequently, results obtained from the full detector arrays will be used for an additional iteration in the detector's design. This is expected to result in a fine tune-up of $\mathrm{G}\left(T_{c}\right), \mathrm{P}_{\text {sat }}$, loop-gain, time-constant and other detector characteristics before arrays are deployed for sky observation.

\section{SUMMARY}

We discussed the design and microfabrication of large arrays of dual-polarization antennacoupled multichroic TES bolometer detectors for cosmic microwave background measurements. These detectors will be used for the third generation camera of the South Pole Telescope, SPT3G. A detailed description of the process involved in the fabrication of the detectors' elements was provided. This includes the development of low-loss $\mathrm{Nb}$ microstrip transmission line to bring the millimeter-wave signal from the antenna to the bolometers. Cross-over structures were also developed, allowing us to route the microstrip and the read out wiring to and from the bolometers. Deployment of the SPT-3G receiver to the South Pole is planned for the end of 2015, with first light expected by the beginning of 2016. Cryogenic optical and noise characterization of the fabricated detectors is still ongoing.

\section{ACKNOWLEDGEMENTS}

This work was supported in part by the Office of Science and the Office of Basic Energy Sciences of the U.S. Department of Energy under Contract DE-AC02- 06CH11357; by the National Science Foundation (NSF) under Grant ANT- 0638937; by the NSF Physics Frontiers Center under Grant PHY-1125897; by The Kavli Foundation; by the Gordon and Betty Moore Foundation; and by the NSF under Grants AST-0956135 and AST-1402161. Technical support from the Nanofabrication Group at the Center for Nanoscale Materials, Argonne National Laboratory, is gratefully appreciated.

\section{REFERENCES}

[1] Penzias, A A and Wilson, R W, 1965 Astrophys. J. 142 419-21.

[2] Dicke, R H, Peebles, P J E, Roll, P G and Wilkinson D T, 1965 Astrophys. J. 142 414-19.

[3] Peebles, P J E, Schramm D N, Turner E L and Kron R G, 1991 Nature 352 769-76.

[4] Smoot, G F, et al. 1992, Astrophys. J. 396 L1-L5.

[5] Hinshaw, G, et al. 2013 ApJS 20819.

[6] Story, et al. 2013 Astrophys. J. 77986.

[7] Sievers, J L, et al. 2013 J. Cosmol. Astropart. Phys. 1310060.

[8] Ade, P A R, et al. $2014 A \& A 571$ A16.

[9] Irwin, K D, 1995 App. Phys. Lett. 66 1998-2000. 
[10] Lee, A T, Richards, P L, Nam, S W, Cabrera B and Irwin K D, 1996 Appl. Phys. Lett. 69 1801-03.

[11] Suzuki, A, et al. 2012 J. Low Temp. Phys. 167 852-58.

[12] Benson, B, et al. 2014 Proc. SPIE 915391531 P.

[13] Bender, A N, Chiche, De Hann, T, Dobbs, M A, Gilbert, A J, Montgomery J, Rowlands N, Smecher G M, Smith K, Wilson A 2014 arXiv:1407.3161.

[14] Suzuki, et al. 2014 J. Low Temp. Phys. 176 650-56.

[15] O’Brient R, et al. 2013. Appl. Phys. Lett. 102063506.

[16] Yefremenko, V, et. al. 2013 IEEE Trans. Appl. Supercond. 232100605.

[17] Wang, G, Yefremenko, V, Chang, C L, Novosad, V, Mehl, J, Pearson, J, Divan, R and Carlstrom, J E, 2013 IEEE Trans. Appl. Supercond. 232101605.

[18] Duhamel, R H, 1987, United States Patent US4658262 A.

[19] Edwards, J M, O'Brient, R, Lee, A T and Rebeiz, G M, 2012 IEEE Trans. Antennas Propag. 60 4082-91.

[20] Lueker M, et al. 2009 IEEE Trans. Appl. Supercond. 19 496-500.

[21] George E. M, et al. 2014 J. Low Temp. Phys. 176383 -91.

[22] O'Brient, R C, 2010 “A log periodic focal-plane architecture for Cosmic Microwave Background Polarimetry,” PhD Dissertation, University of California, Berkeley.

[23] Suzuki, A, 2013 "Multichroic Bolometric Detector Architecture for Cosmic Microwave Background Polarimetry Experiments," PhD Dissertation, University of California, Berkeley.

[24] Chang, C L, et al. 2015 IEEE Trans. Appl. Supercond. 25 (2015).

[25] Maxfield, B W and McLean, W L 1965 Phys. Rev. 139 A1515-A1522.

[26] Kerr, A 1999 Surface impedance of superconductors and normal conductors in EM simulators (1999). Available online at http://legacy.nrao.edu/alma $/ \mathrm{memos} / \mathrm{html}$ memos/alma245/memo245.pdf.

[27] Kumar, S, Vayonakis, A, LeDuc, H, Day, P, Golwala, S and Zmuidzinas, J, 2009 IEEE Trans. Appl. Supercond. 19 924-929.

[28] Fujimoto, R, et al, 2000 Nucl. Instrum. Meth. A 444 180-83.

[29] Sadleir, J E, Smith, S J, Bandler, S R, Chervenak, J A and Clem, J R, 2010 Phys. Rev. Lett. 104047003.

[30] Sadleir, J E, Smith S J, Robinson, I K, Finkbeiner, F M, Chervenak, J A, Bandler, S R, Eckart M E and Kilbourne C A, 2011 Phys. Rev. B 84184502.

[31] L. Gottardi, et al, 2014 Appl. Phys. Lett. 105162605. 